\title{
Molecular features in a biphenotypic small cell sarcoma with neuroectodermal and muscle differentiation
}

Enrique de Alava, Md, Phd, María Dolores Lozano, Md, Phd, Iosu Sola, Md, Phd, Angel Panizo, Md, Phd, Miguel A. Idoate, Md, Phd, Carmen Martínez-Isla, Md, Phd, Jerónimo Forteza, Md, Phd, Luis Sierrasesúmaga, Md, Phd, FRCF, and F. Javier Pardo-Mindán, Md, Phd

From the Departments of Pathology and Pediatrics, Clínica Universitaria de Navarra, Pamplona, Spain, and the Department of Pathology, Universidade de Santiago de Compostela, Santiago de Compostela, Spain.

Supported by a grant of the Regional Government of Navarra 2246/96, Pamplona, Spain, and a grant of the Public Health Care System of Spain FISS 96/2102, Spain.

Address correspondence and reprint requests to Enrique de Alava, MD, PhD, Departamento de Anatomía Patológica, Clínica Universitaria de Navarra, Apartado 4209, 31080 Pamplona, Spain.

\begin{abstract}
We report a case of a 13-year-old girl with soft tissue sarcoma of the hand, which showed muscle and neuroectodermal immunophenotypes. Molecular studies were performed on RNA collected from fine-needle aspiration (FNA) cytology and peripheral blood samples by nested reverse transcriptase-polymerase chain reaction (RT-PCR) and Southern blot analysis. This biphenotypic tumor showed simultaneous expression of EWS-FLI1 and PAX3-FKHR transcripts, specific of Ewing family tumors and alveolar rhabdomyosarcoma, respectively. Although childhood sarcomas with simultaneous muscle and neural differentiation have been described to have EWS-FLI1 transcripts, there are no reports of tumors with both transcripts. Cytological specimens are a good source of RNA for molecular studies.
\end{abstract}

\section{KEY WORDS}

Malignant ectomesenchymoma, Ewing sarcoma/PNET, alveolar rhabdomyosarcoma, EWS-FLI1 chimeric transcript, PAX3-FKHR chimeric transcript.

\section{ABBREVIATIONS}

RT-PCR, reverse transcription-polymerase chain reaction; FNA, fine-needle aspiration cytology; PNET, peripheral neuroectodermal tumor; MRI, magnetic resonance imaging; PBS, peripheral blood sample. 


\section{INTRODUCTION}

Small round cell tumors of childhood remain a diagnostic challenge to the surgical pathologist because they share several morphological features and are often difficult to distinguish from each other. Because effective therapeutic protocols are available, an accurate diagnosis is needed to institute appropriate therapy and predict prognosis. Consistent cytogenetic alterations have been identified in several members of this family, including alveolar rhabdomyosarcoma, Ewing's sarcoma/peripheral neuroectodermal tumor (PNET), and desmoplastic small round cell tumor. ${ }^{1}$ Recently, these chromosomal translocations have been characterized at the molecular level and found to result in PAX3-FKHR or PAX7-FKHR chimeric genes in alveolar rhabdomyosarcoma, ${ }^{2}$ EWS-FLI1 and EWS-ERG gene fusions in Ewing's sarcoma/ PNET, ${ }^{3}$ and EWS-WT1 gene fusions in desmoplastic small round cell tumor (DSRCT) ${ }^{4}$ Chimeric transcripts and gene fusions specific for each translocation can be detected in clinical samples by reverse transcriptase-polymerase chain reaction (RT-PCR) and fluorescent in situ hybridization (FISH), and are proving to be a reliable and very useful diagnostic technique in the small round cell tumor family. ${ }^{1,5}$

In this case study, we apply these molecular assays to detect chimeric transcripts in cytological specimens and peripheral blood samples in a sarcoma with neuroectodermal and muscle differentiation.

\section{CASE REPORT}

A 13-year-old girl developed a painful soft tissue mass in the hypothenar region of the left hand. A magnetic resonance imaging (MRI) scan showed a poorly circumscribed mass, $2 \mathrm{~cm}$ in diameter, without bone involvement. An open biopsy was performed. It disclosed (Fig 1A) an undifferentiated solid tumor made up of strands of small cells with hyperchromatic nuclei and scant cytoplasm, with material that tested positively with periodic acid-Schiff (PAS). The patient was referred to our hospital under the diagnosis of extraskeletal Ewing's sarcoma.

Once admitted in our hospital, a left axillary mass was detected. FNAs were performed in both the axilla and the left hand mass. They showed the same cytological picture, including small cells with hyperchromatic nuclei, sometimes eccentric, finely stippled chromatin, occasional nucleoli, and a scant eosinophilic cytoplasm with a small number of multinucleated cells were seen. An immunohistochemical study was performed on the FNA smears of both locations with antibodies for pan-cytokeratins, vimentin, desmin, S-100 protein, NSE, and O13. Cytoplasmic immunoreactivity was detected for vimentin, S-100 protein, NSE, O13 (Fig 1B), and desmin (Fig 1C).

A treatment program following the protocol for highgrade sarcoma, including radiotherapy and high-dose chemotherapy, was administered. Two months later, the axillary mass was resected. It corresponded to a lymph node in which necrotic tumor nests were seen in a fibrotic background. A small number of viable tumor cells was seen with features similar to those in previous samples (Fig 1D). The patient was discharged in good status and is currently finishing the treatment protocol with no gross evidence of disease. 


\section{MOLECULAR STUDIES}

Available samples for molecular studies include in chronological order: FNAs of the left hand mass and lymph node, lymph node excision, and four peripheral blood samples (PBS). The first was taken 2 weeks after the FNAs, and the remaining samples on a monthly basis.

RNA extraction was performed immediately after the samples were taken with a modified guanidine isothiocyanate method, using the reagent TRIZOL (Gibco BRL). Search for chimeric transcripta was then performed by nested RT-PCR using $1 \mu \mathrm{g}$ of total RNA as template and a GeneAmp RNA-PCR kit (Perkin-Elmer, Roche Molecular Systems, Branchburg, NJ) using the manufacturer's recommendations. Reverse transcription included an incubation period of 45 minutes at $42^{\circ} \mathrm{C}$ with random hexamers, followed by a 5 -minute period at $99^{\circ} \mathrm{C}$ to denature the enzyme. PCR was then performed with an initial denaturation step of $95^{\circ} \mathrm{C}$ for 2 minutes, followed by 35 cycles including a denaturation step at $94^{\circ} \mathrm{C}$ for 30 seconds, annealing at $66^{\circ} \mathrm{C}\left(68^{\circ} \mathrm{C}\right.$ for the second round) for 30 seconds, and extension at $72^{\circ} \mathrm{C}$ for 60 seconds, with 7 minutes of extension after the completion of the last cycle. A second round of amplification was performed in PBS. One fifth of the initial PCR product was amplified with a second set of specific internal primers. All PCR products were identified by $2 \%$ agarose gel electrophoresis and ethidium bromide staining, and then blotted into nylon membranes (Hybond+; Nycomed Amersham, Buckinghamshire, United Kingdom), and detected with internal probes labeled with chemoluminiscence (Nycomed Amersham). Specific primers and probes were used to amplify EWS-FLI1, EWS-ERG, PAX3FKHR, and EWS-WT1 and have been reported elsewhere. ${ }^{5,6}$

EWS-FLI1 chimeric transcripts were detected in FNA but not in PBS. The length of the amplified product was constant (Fig 2). Sequencing of the RT-PCR product revealed an in-frame fusion of EWS exon 7 to FLIl exon 6, 329 base pairs in length. PAX3-FKHR chimeric transcripts (Fig 3) were present in the FNAs performed on the hand mass and on the axillary lymph node. They were not present, however, in the lymph node excision, 2 months later. EWS-ERG and EWS-WT1 transcripts were not found in any sample.

\section{DISCUSSION}

Malignant ectomesenchymomas are sarcomas where areas of neural and muscle differentiation are seen. Its primitive variants are composed of undifferentiated cells, resembling peripheral neuroepithelioma in some areas and alveolar rhabdomyosarcoma in others. ${ }^{7}$ Our results show a soft tissue tumor in which a double differentiation occurred. Immunohistochemical studies support both neuroectodermal and muscle differentiation. Moreover, simultaneous expression of EWS-FLI1 and PAX3-FKHR fusion transcripts could be shown in this tumor. Those gene fusions are considered specific for Ewing family tumors and alveolar rhabdomyosarcomas, respectively. Reports in which simultaneous investigation of several chimeric transcripts have been performed in small round cell tumors have shown a high correlation between histopathological entities and chimeric transcripts. ${ }^{4,5}$ 
EWS-FLI1 chimeric transcripts have been found in undifferentiated tumors that have morphology that is not consistent with ES/PNET, such as the cases reported by Thorner et $\mathrm{al}^{8}$ or an intraabdominal small round cell tumor with morphological features of a DSRCT. ${ }^{9}$ None of these studies, however, report the simultaneous finding of two chimeric transcripts in a given tumor. EWS-FLI1 transcripts were also reported in a series of childhood malignant ectomesenchymomas. ${ }^{10}$ The authors described six biphenotypic sarcomas with both neural and muscle differentiation appearing in soft tissues of the chest, the extremities, or retroperitoneum in patients between the ages of 5 months and 25 years. Interestingly, all these tumors expressed EWS-FLI1 transcripts identical to those of the Ewing family of tumors. None of the cases expressed any other transcript, including PAX3-FKHR. In another study, however, one of two ectomesenchymomas studied showed the PAX3-FKHR fusion gene, which is associated with alveolar rhabdomyosarcomas, without evidente of EWS-FLI1 transcripts. ${ }^{11} \mathrm{~A}$ primitive ectomesenchymoma cell fine has been reported to have the $t(11 ; 22)$ (q24;q12) associated with the Ewing family of tumors and another rearrangement involving the band 13q14, one of the breakpoints of the $\mathrm{t}(2 ; 13)$ ( $\mathrm{q} 35$; q14) translocation of alveolar rhabdomyosarcoma, which was not present. ${ }^{12}$ Our case is unique for being a primitive malignant ectomesenchymoma expressing both transcripts.

FNA has been used in small round cell childhood tumors to obtain material suitable to perform cytogenetic studies, ${ }^{13}$ but karyotypes are laborious and often unsuccessful. As far as we know, this is the second report in which EWS-FLI1 transcripts have been found on cytological specimens, ${ }^{13}$ and the first in which PAX3-FKHR fusions are detected in FNA material. Our study indicates that material obtained from FNAs, if immediately processed, is probably an excellent source of RNA for molecular studies. RT-PCR is sensitive enough to detect chimeric transcripts in small samples of aspirated tissue.

The high sensitivity of RT-PCR permits the detection of fusion RNAs in peripheral blood samples, a promising assay to detect circulating tumor cells and minimal residual disease in undifferentiated sarcomas of childhood. It is being used in patients with Ewing family tumors ${ }^{6}$ and alveolar rhabdomyosarcomas ${ }^{14}$ as a possible means to monitor therapy. In our case, it allowed us to monitor the presence of EWS-FLI1 transcripts in the patient while she was undergoing treatment in which all PBS are negative for transcripts. However, the possible prognostic value of chimeric RNA detection in childhood sarcomas is still unclear.

The expression of two transcripts seems to be an exceptional feature in childhood tumors. Two possible explanations include primitive cells capable of differentiating simultaneously along two different pathways, or one of these two transcripts could be a secondary genetic alteration on an otherwise conventional alveolar rhabdomyosarcoma or Ewing family tumor. Tumors with divergent differentiation such as desmoplastic small round cell tumor are a good example of the first possibility, and a challenge to tumor classification. ${ }^{5}$ Because secondary molecular features in pediatric tumors have to be further described, the second possibility cannot be discarded. Regardless of etiology, this case has shown that neither morphology or our current molecular knowledge are sufficient to classify rare cases of childhood solid tumors. 


\section{ACKNOWLEDGMENT}

We thank Loli Martínez for expert technical help in the immunohistochemical procedures, and Javier Gardeta for photographic assistance.

\section{NOTE ADDED IN PROOF}

Two months after finishing therapy the patient presented progression involving lung and multiple bone foci. PBS at this time were positive for EWS-FLI1 transcripts. The patient died of disease 4 months after completion of therapy.

\section{REFERENCES}

1. Barr FG, Chatten J, D' Cruz CM, et al: Molecular assays for chromosomal translocations in the diagnosis of pediatric soft tissue tumors. JAMA 273:553-557, 1995

2. Barr FG, Galili N, Holick J, et al: Rearrangement of the PAX3 paired hoz gene in the paediatric solid tumour alveolar rhabdomyosarcoma. Nature Genetics 3:113-117, 1993

3. Delattre O, Zucman J, Melot T, et al: The Ewing family of tumors. A subgroup of small round cell tumors defined by specific chimeric transcripts. N Engl J Med 331:294-299, 1994

4. Ladanyi M, Gerald W: Fusion of the EWS and WT1 genes in the desmoplastic small round cell tumor. Cancer Res 54:2837-2840, 1994

5. de Alava E, Ladanyi M, Rosai J, Gerald WL: Detection of chimeric transcripta in desmoplastic small round cell tumor and related developmental tumors by RT-PCR. A specific diagnostic assay. Am J Pathol 147:15841591, 1995

6. Pfleiderer C, Zoubek A, Gruber B, et al: Detection of tumour cells in peripheral blood and bone marrow from Ewing tumour patients by RT-PCR. Int J Cancer 64:135-139, 1995

7. Parham DM: Ewing's sarcoma and related tumors, in Parham D: Pediatric Neoplasia. Morphology and Biology. Philadelphia, PA, Lippincott-Rayen, 1996, pp 65-85

8. Thorner P, Squire J, Chilton-McNeill S, et al: Is the EWS/FLI-1 fusion transcript specific for Ewing sarcoma and peripheral primitive neuroectodermal tumor? A report of four cases showing this transcript in a wider range of tumor types. Am J Pathol 148:1125-1138, 1996

9. Katz RL, Quezado M, Senderowicz AM, et al: An intraabdominal small round cell neoplasm with features of primitive neuroectodermal and desmoplastic round cell tumor and a EWS-FLI1 fusion transcript. Hum PATHOL 28:502-509, 1997

10. Sorensen PHB, Shimada H, Liu XF, et al: Biphenotypic sarcomas with myogenic and neural differentiation express the Ewing's sarcoma EWS/FLI1 fusion gene. Cancer Res 55:1385-1392, 1995

11. Downing JR, Khandekar A. Shurdeff SA, et al: Multiplex RT-PCR assay for the differential diagnosis of alveolar rhabdomyosarcoma and Ewing's sarcoma. Am J Pathol 146:626-634, 1995 
12. Whang-Peng J, Knutsen $\mathrm{T}$, Theil $\mathrm{K}$, et al: Cytogenetic studies in subgroups of rhabdomyosarcoma. Genes Chromosomes Cancer 5:299-310, 1992

13. Akerman M, Dreinhofer E, Rydholm A, et al: Cytogenetic studies on fineneedle aspiration samples from osteosarcoma and Ewing's sarcoma. Diagn Cytopathol 15:17-22, 1996

14. Kelly KM, Womer RB, Barr FG: Minimal residual detection in alveolar rhabdomyosarcoma using a reverse transcriptase-polymerase chain reaction. Cancer 78:1320-1327, 1996 

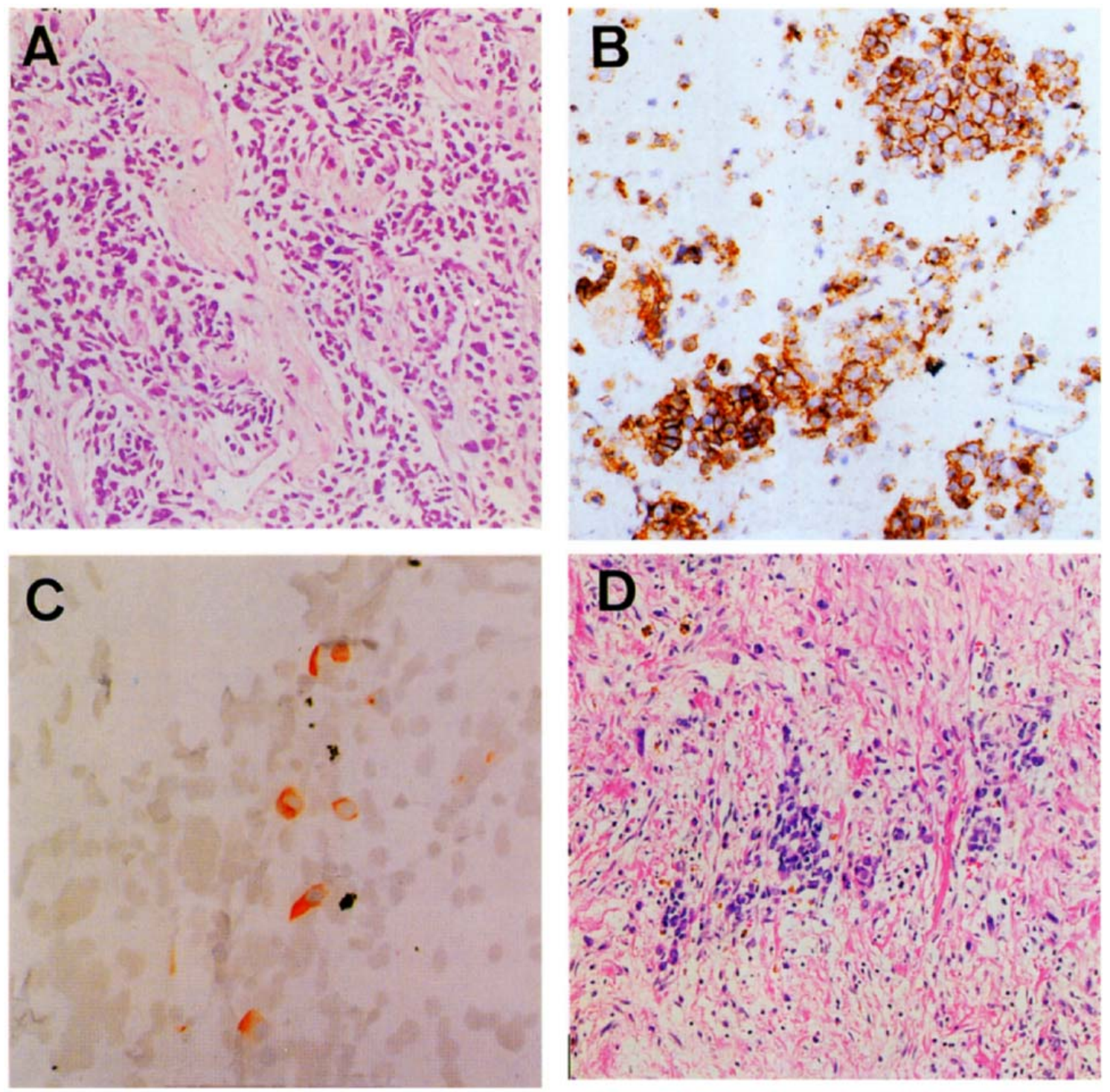

Figure 1. (A) Primary tumor. Strands of small cells with scanty cytoplasm and hyperchromatic nuclei (H\&E stain; original magnification $\times 100)$. (B) FNA of the axillary mass showing intense membrane immunoreactivity for $\mathrm{O} 13$ (Streptavidine biotin peroxidase counterstained with hematoxylin; original magnification $\times 100$ ). (C) FNA of the axillary mass; tumor ceils show cytoplasmic immunoreactivity for desmin (Streptavidine biotin peroxidase counterstained with hematoxylin; original magnification $\times 150$ ). (D) Axillary mass resection. Only a few tumor nests remain after treatment in an otherwise necrotic lymph node (H\&E stain; original magnification $\times$ 100). 


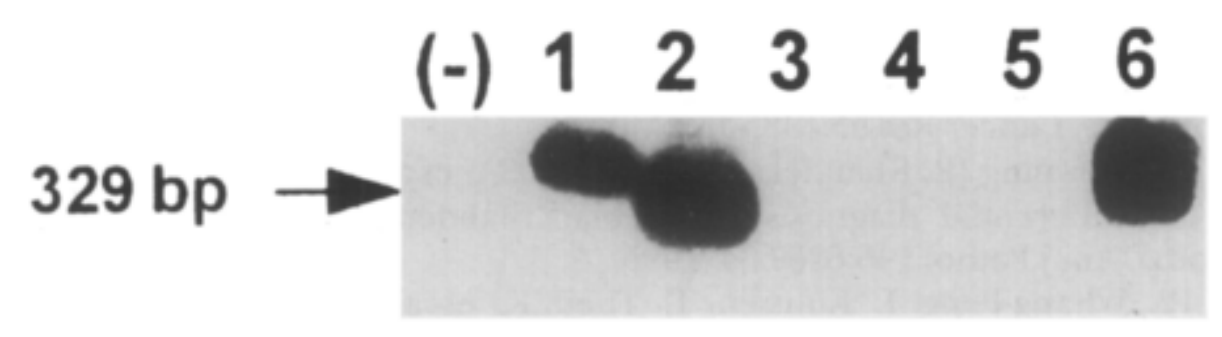

Figure 2. Detection of EWS-FLI1 transcripts by RT-PCR and Southern blot analysis on several patient samples. Expected size of the transcript is 329 bp. Lane 1, FNA of the hand; lane 2, FNA of the axillary mass; lanes 3-5, Peripheral blood samples taken 2 weeks, 2 months, and 3 months after resection of the axillary mass, respectively; lane 6 , positive control.

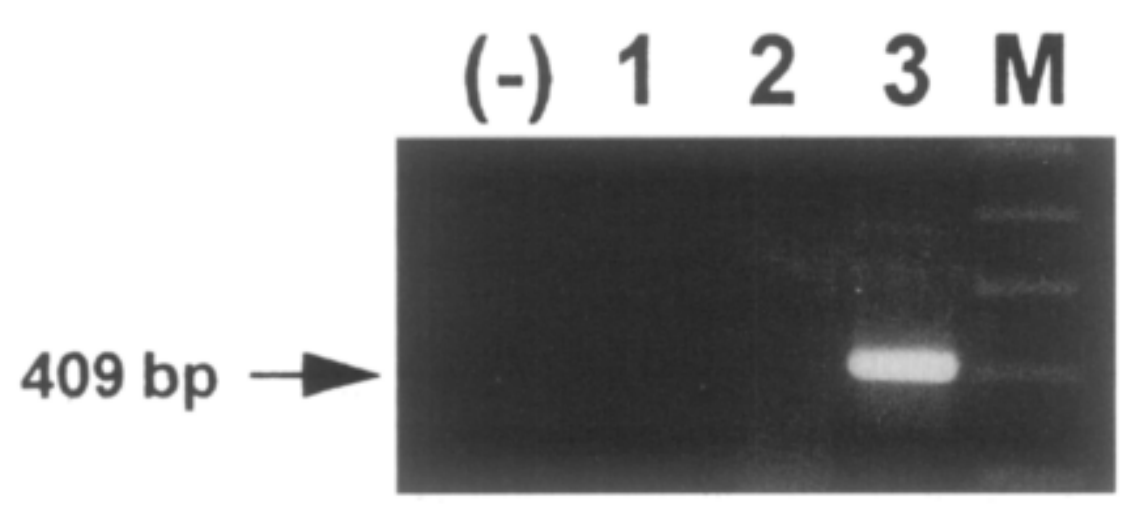

Figure 3. Detection of PAX3-FKHR transcripts (409 bp) by RT-PCR. Lane 1, Ewing's sarcoma cell line; lane 2, resection of the axillary mass; lane 3, FNA of the axillary mass. 